\title{
Comprehensive Weighted Clique Degree Ranking Algorithms and Evolutionary Model of Complex Network
}

\author{
Jie $\mathrm{Xu}^{1, *}$, Zhen $\mathrm{Liu}^{1}$, and Jun $\mathrm{Xu}{ }^{1}$ \\ ${ }^{1}$ Key Lab of Optical Fiber Sensing and Communications (Ministry of Education), School of Communication and Information Engineering, \\ University of Electronic Science and Technology of China, No.2006, Xiyuan Ave, Chengdu 622731,China
}

\begin{abstract}
This paper analyses the degree ranking (DR) algorithm, and proposes a new comprehensive weighted clique degree ranking (CWCDR) algorithms for ranking importance of nodes in complex network. Simulation results show that CWCDR algorithms not only can overcome the limitation of degree ranking algorithm, but also can find important nodes in complex networks more precisely and effectively. To the shortage of small-world model and BA model, this paper proposes an evolutionary model of complex network based on CWCDR algorithms, named CWCDR model. Simulation results show that the CWCDR model accords with power-law distribution. And compare with the BA model, this model has better average shortest path length, and clustering coefficient. Therefore, the CWCDR model is more consistent with the real network.
\end{abstract}

\section{Introduction}

In recent years, research of complex networks ${ }^{[1-2]}$ become the hot topic in network research. More and more fields involve the research of complex network, such as biology, economics, sociology, etc ${ }^{[3]}$. Research of important nodes ranking $^{[4-5]}$ and evolutionary model of complex network have become more and more important in network research. Important nodes ranking is always based on degree, betweenness, closeness, etc. These algorithms have different preference characteristics, so different algorithms may reach different results of importance ranking of the nodes ${ }^{[6]}$. These typical nodes ranking algorithms have their own defect. ZHOU proposes the concept of clique degree in his paper ${ }^{[7]}$. This concept considers the close degree between neighbor nodes, and provides a new way for nodes ranking.

Clique degree can be a characterization which can represent the close degree between the nodes, and the clique equals the concept complete subgraph in graph theory. That means $m$-order equal a fully connected network with $m$ nodes and $\mathrm{m}(\mathrm{m}-1) / 2$ edges. Define the $m$ clique degree of a node $i$ as the number of defferent $m$ cliques containing $i$, denoted by $k_{i}^{m}$. Obviously, a 2clique is an edge and $k_{i}^{2}$ equals the degree $k_{i}$. So clique degree can be considered as an extension of degree. We calculated the clique degree of node-4 from 2-order to 5order for representative networks, $k_{4}^{2}=7, k_{4}^{3}=5, k_{4}^{4}=1$, $k_{4}^{5}=0$, as shown in Figure 1.

Clique degree partly reflects the node degree, and it considers the node location in the network. So we propose a new important node ranking algorithms in complex networks base on clique degree concept, named comprehensive weighted clique degree ranking (CWCDR) algorithm.

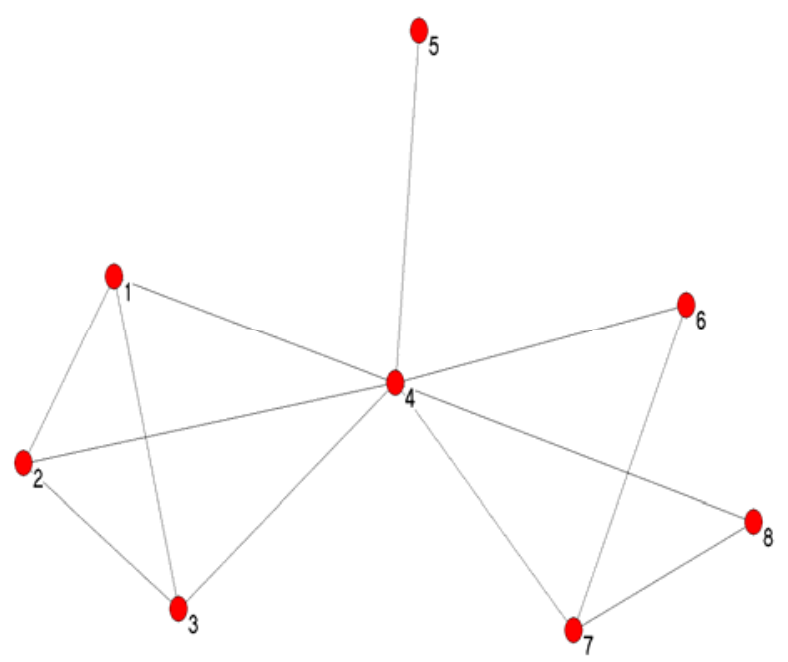

Figure 1. Clique degree of network

\section{Comprehensive weighted clique degree algorithm}

Comprehensive weighted clique degree algorithm introduces four parameters: $\alpha, \beta, \gamma, \lambda$, and four characteristic quantity: 2 -order to 5 -order clique degree. ZHOU indicates that the higher order in clique degree no longer has the strict power-law distribution in his paper ${ }^{[7]}$. According to the theory of six degrees of separation, people can find anyone through six sides in human social

*Corresponding author: xuj@uestc.edu.cn 
networks. So we do not consider above 6-order clique degrees. Thus, the equation of algorithm is as follows:

$$
N(i)=\alpha N^{2}(i)+\beta N^{3}(i)+\gamma N^{4}(i)+\lambda N^{5}(i)
$$

Where $N^{j}(\mathrm{i})$ represents $j$-order clique degree, $N(\mathrm{i})$ represents multi-order comprehensive weighted degree, which is the measurement of nodes ranking. Then we talk about how to get value of $\alpha, \beta, \gamma, \lambda$.

$$
N(i)=\left\{\begin{array}{l}
N^{2}(i)(\alpha=1, \beta=0, \gamma=0, \lambda=0) \\
N^{3}(i)(\alpha=0, \beta=1, \gamma=0, \lambda=0) \\
N^{4}(i)(\alpha=0, \beta=0, \gamma=1, \lambda=0) \\
N^{5}(i)(\alpha=0, \beta=0, \gamma=0, \lambda=1) \\
\alpha N^{2}(i)+\beta N^{3}(i)+\gamma N^{4}(i)+\lambda N^{5}(i), \text { others }
\end{array}\right.
$$

Where $\alpha+\beta+\gamma+\lambda=1$, assuming $m$ as the 2 -order clique degree, $n$ as the 3 -order clique degree, $k$ as the 4order clique degree, and $t$ as the 5-order clique degree. By normalization of $m, n, k, t$, we get $p(m), p(n), p(k), p(t)$. Their reciprocals are as follows:

$$
\frac{1}{p(m)}, \frac{1}{p(n)}, \frac{1}{p(k)}, \frac{1}{p(t)}
$$

Comprehensive weighted clique degree considers the closeness level among nodes in the network. It measures the effect of each order clique degree in important nodes ranking by normalization. The higher order clique degree in the network is more important. So we get the results of $\alpha, \beta, \gamma, \lambda$ as follows:

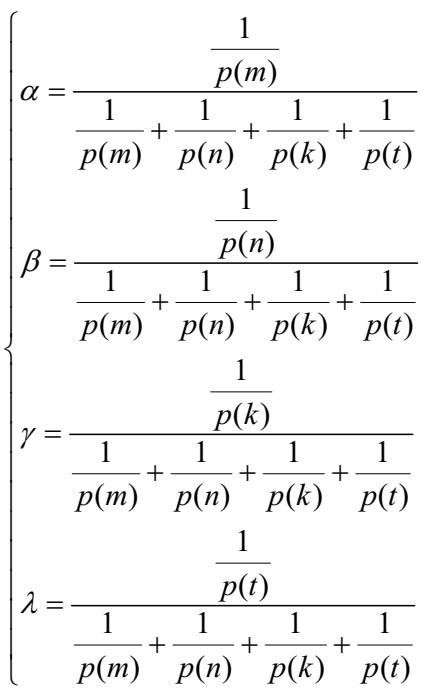

\section{Analysis of algorithm}

Karate club network is the most popular real network data model in complex network research. This network is composed of 34 people. Then this network is abstracted to the graph theory model. This model contains 34 nodes, and every edge between two nodes denotes the two members have strong social relationships in real world. This network model is as follows in Figure 2.

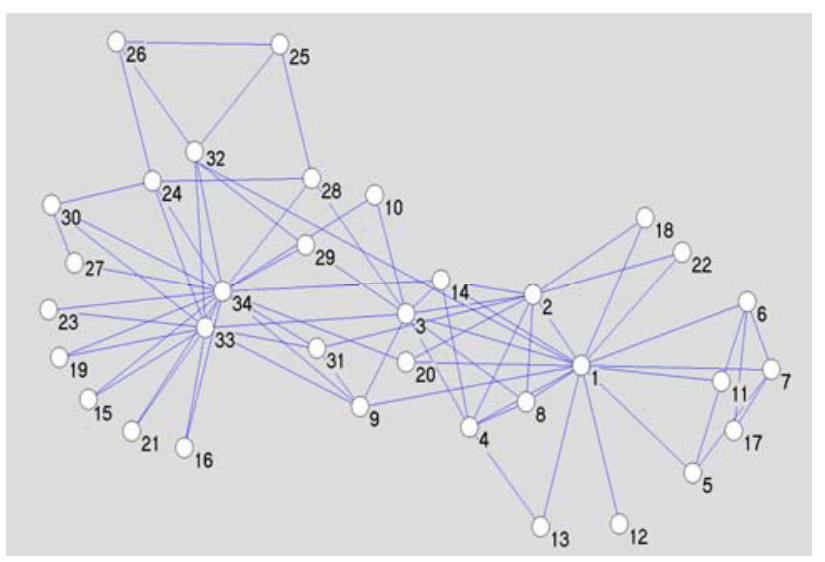

Figure 2. Karate club network

We know that the importance of the nodes in the margin is relatively low through Figure 2 . In order to verify that our algorithm is better than degree ranking (DR) algorithm, closeness degree ranking (CDR) algorithm, betweenness ranking (BR) algorithm and PageRank ranking (PR) algorithm. We get the ranking results through the comprehensive weighted clique degree ranking (CWCDR) algorithm, and then we compare the results with these typical algorithms in Table 1 .

Table 1. The comparison between the algorithms

\begin{tabular}{|c|c|c|c|c|c|c|c|c|c|}
\hline ID & DR & ID & BR & ID & CDR & ID & PR & ID & $\begin{array}{c}\text { CW } \\
\text { CDR }\end{array}$ \\
\hline 34 & 17 & 1 & 0.8238 & 1 & 0.0172 & 34 & 0.1009 & 1 & 3.67 \\
\hline 1 & 16 & 34 & 0.5724 & 3 & 0.0169 & 1 & 0.0970 & 3 & 3.37 \\
\hline 33 & 12 & 33 & 0.2734 & 34 & 0.0167 & 33 & 0.0717 & 2 & 3.33 \\
\hline 3 & 10 & 3 & 0.2704 & 32 & 0.0164 & 3 & 0.0571 & 4 & 3.11 \\
\hline 2 & 9 & 32 & 0.2603 & 33 & 0.0156 & 2 & 0.0529 & 8 & 2.01 \\
\hline 32 & 6 & 9 & 0.1053 & 14 & 0.0156 & 32 & 0.0372 & 34 & 1.87 \\
\hline 4 & 6 & 2 & 0.1015 & 9 & 0.0156 & 4 & 0.0359 & 33 & 1.49 \\
\hline 24 & 5 & 14 & 0.0863 & 20 & 0.0152 & 24 & 0.0315 & 14 & 0.44 \\
\hline 9 & 5 & 20 & 0.0611 & 2 & 0.0147 & 9 & 0.0298 & 30 & 0.44 \\
\hline 14 & 5 & 6 & 0.0564 & 4 & 0.0141 & 14 & 0.0295 & 9 & 0.38 \\
\hline
\end{tabular}

In the Table 1, we can see in BR algorithm ranking, Node- 6 is the tenth in the top 10 ranking, and Node- 4 cannot rank in top 10. It's mean that Node-6 is more important than Node- 4 in this algorithm. But it is easy to see in Figure 2 that Node- 4 is in the central position of the network, and it is the bridge for Node-1, Node-2, Node-3, and Node-14, so we think in fact Node-4 is more important than Node-6. Thus, the CWCDR presents this result better as shown in Table 1. On the other hand, CDR algorithm cannot distinguish the importance of the nodes in the central position of the network, such as Node-32 and Node- 8 . The CDR value of Node-32 is much bigger than Node- 8 (Node- 8 cannot rank in top 10 in Table 2), but we find that the network cannot operate without Node- 8 in reality and can operate without Node32. So we think Node- 8 is more important than Node-32.

In Table 1, PR algorithm obtains Node-32 is more important than Node-4. However, Node-4 is the core node of right sub-network, and it plays the role bridge connecting two networks. Node-4 also has close relationship with Node-1, Node-2, Node-14, which are at very important positions in the network. Although Node32 has the function bridge connecting, it is far from core 
positions of two sub-networks. So we obtain Node-4 is more important than Node-32, and CWCDR presents this result well.

\section{The evolutionary network model based on CWCDR algorithm}

\subsection{The evolutionary network model}

Real networks have scale-free network characteristics, and BA is a typical model of scale-free networks. So we choose growth properties and preferential connection properties of BA evolutionary network model to evolve the model based on CWCDR algorithms ${ }^{[8-10]}$, the evolutionary algorithms is as follows:

i) Original Network: the original network has $n$ nodes, and we calculate the CWCDR value through CWCDR algorithm.

ii) Growth : a new node will join in the network every once in a while, we choose $m$ nodes of the network to connect the new node, where $m<n$.

iii) Preferential connection: the new node $i$ choose $m$ nodes which the CWCDR value is highest in the network to connect. The connection probability $p_{i}$ is:

$$
p_{i}=\frac{N_{i}}{\sum_{j} N_{j}}
$$

iv) Iteration and update: the new node changes the topological connection of the network, so we update the CWCDR value of the nodes.

v) Back: if it has new node to join in the network, back to ii); if not, the algorithm is over. Then the network has $N=n+t$ nodes and $m^{*} t$ edges, where $t$ is the number of new nodes.

\subsection{Simulation and Analysis}

The preferential connection mechanism of BA network evolution model is based on the DR algorithm. But CWCDR algorithm reconstruct the importance ranking of the nodes, and calculate the connection probability based on these ranking values. By the simulation in Matlab, we compare the evolutionary network model based on CWCDR algorithm with the BA model, and analyze degree distribution, average shortest path, and clustering coefficient. The results are in the Figure 3.

We assume the network has 2000 nodes after a period of time, and the first new node connects all the nodes in the network when it joins the network. The original networks have $m_{0}$ nodes, and $m_{0}$ are $2,4,6$, and 8 respectively. When the new nodes join in the network, we choose $m=m_{0}$.

In the Figure 3(a), we know that the evolutionary network model based on CWCDR algorithms obeys power-law distribution. When the number of initial nodes is different, the value of power-law distribution function is the same. We can see that the small degree values nodes are in the majority. Degree value distribution is scattered, and degree distribution in the network is scalefree distribution.

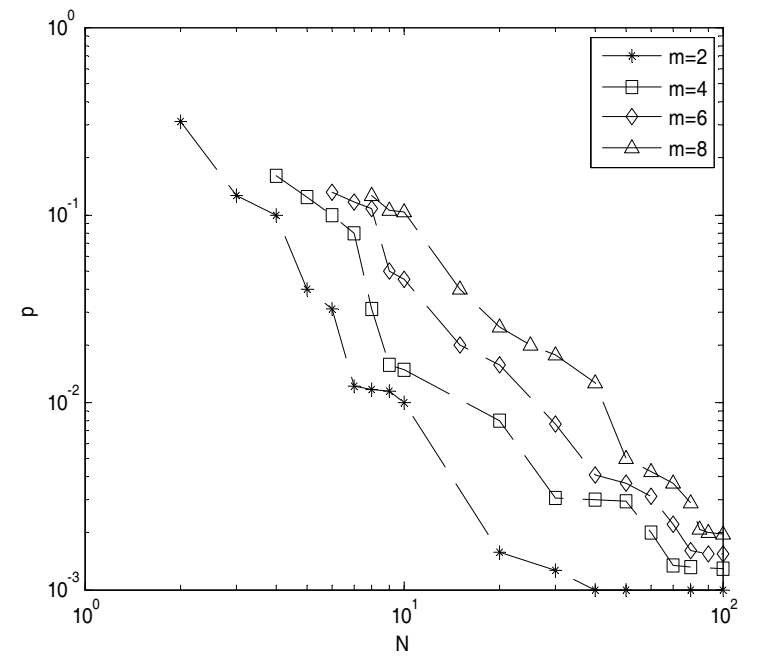

(a) Degree distribution

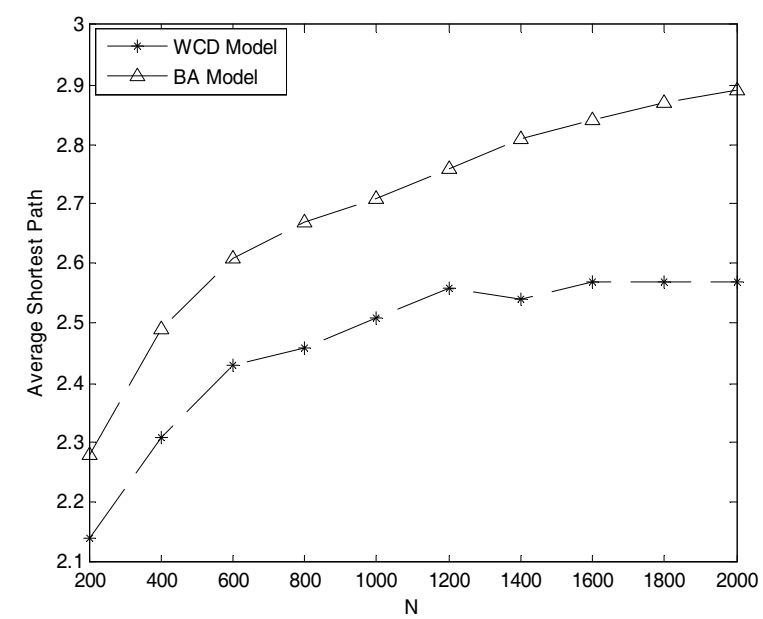

(b) Average shortest path

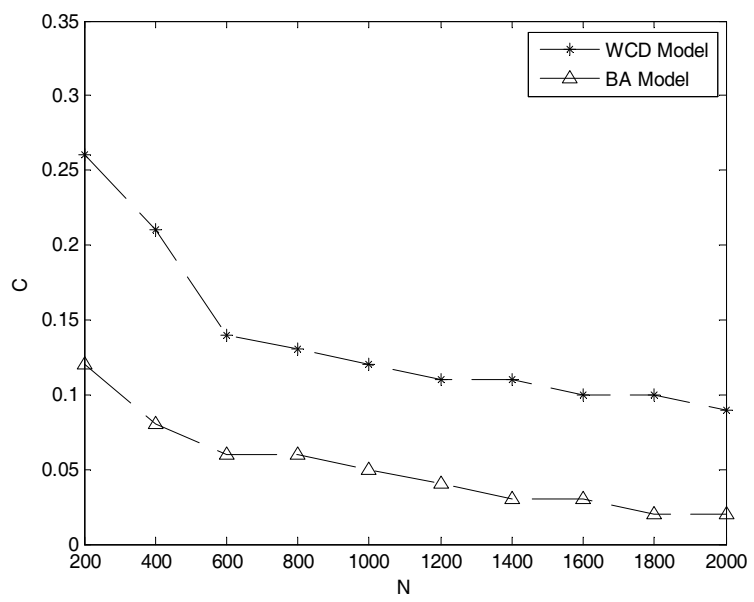

(c) Clustering coefficient

Figure 3. The comparison between our model and BA model

In the Figure 3(b), the average shortest path of our model is smaller than BA model, and it grows slowly 
with the growth of the network size. When the network has more than 2000 nodes, the average shortest path of the mode will remain unchanged. So the model is consistent with the small world network features.

In the Figure 3(c), the clustering coefficient of the model is bigger than BA model. When the network has more than $N_{t}$ nodes, the clustering coefficient of the two models will remain unchanged.

The network in reality always is power-law distribution, and it has big clustering coefficient and small average shortest path. We know BA model consistent with the real network features and the model based on CWCDR algorithms is better than BA model in degree distribution, average shortest path, and clustering coefficient.

\section{Conclusions}

This paper proposes the comprehensive weighted clique degree algorithm based on the clique degree. The algorithm considers not only degree, but also the closeness of nodes with their neighbor nodes. Analyzed some famous real network models, we prove it more accurate than degree ranking algorithm, closeness degree ranking algorithm, betweenness ranking algorithm and PageRank ranking algorithm. Then according to the growth and preferential connection steps of evolutionary model, we propose a new evolutionary network model based on comprehensive weighted clique degree algorithm. Calculate the degree distribution, clustering coefficient and average shortest path of the model, and we compare the result with BA model. The result is better than BA model, which proves it better than BA model to consistent with the real network features.

\section{Acknowledgments}

This work was supported by NSFC Foundation (61201128), Sichuan Province Scientific and Technological Support Project (2014GZ0017, 2016GZ0093), Program for Changjiang Scholars and Innovative Research Team (PCSIRT) in University and the 111 Project B14039, the Fundamental Research Funds for the Central Universities (ZYGX2015J009).

\section{References}

1. S.H. Strogatz, Exploring complex networks. Nature, 410, 268-276 (2001)

2. R. Albert, A.L. Barabasi, Statistical mechanic of complex networks. Rev.Mod.Phys, 74, 1-54 (2002)

3. G.X. Wang, Y. Shen, E.J. Luan, A measure of centrality based on modularity matrix, Progress in Nature Science, 18, 1043-1047 (2008)

4. J. Wu, Y.J. Tan, Finding the Most Vital Node by Node Contraction in Communication Networks. International Conference on Communications, Circuits and Systems, 2 (2005)
5. D.C. Huang, H.C. Qi, PageRank Algorithm Research. Computer Engineering, 32, 145-146 (2006)

6. X.P. Zhang, Y.S. Li, Evaluation Method of Importance for Nodes in Complex Networks Based on Importance Contribution. Comlplex Systems And Complexity Science, 11, 25-26 (2014)

7. W.K. Xiao, T. Zhou, Empirical study on cliquedegree distribution of networks. PHYSICAL REVIEW E, 76, 1-4 (2007)

8. J.R. Li, A Node Centrality Evaluation Model for Weighted Social Networks. Journal of University of Electronic Science and Technology of China, $\mathbf{4 3}$ (2014)

9. X. Li, G.R. Chen, A local-world evolving network model. Physical A: Theoretical and Statistical Physics, 328, 274-286 (2003)

10. X.L. Wang, P2P network node importance measurement method based on multi-attribute. Journal of Computer Applications, 34, 7-10 (2014) 